\title{
Disturbance rejection using an ILC algorithm with iteration varying filters
}

\author{
Mikael Norrlöf \\ Division of Automatic Control \\ Department of Electrical Engineering \\ Linköpings universitet, SE-581 83 Linköping, Sweden \\ WWW: http://WwW.control.isy.liu.se \\ E-mail: mino@isy.liu.se
}

6th February 2004

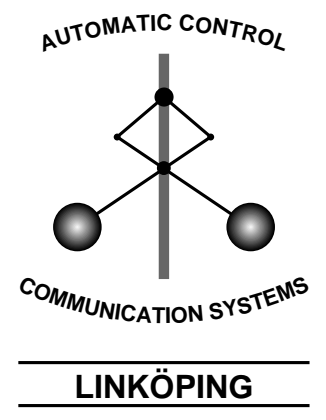

Report no.: LiTH-ISY-R-2588

Submitted to Asian Journal of Control (Vol.6, No. 3)

Technical reports from the Control \& Communication group in Linköping are available at http://www.control.isy.liu.se/publications. 


\begin{abstract}
An Iterative Learning Control disturbance rejection approach is considered and it is shown that iteration variant learning filters can asymptotically give the controlled variable zero error and zero variance. Convergence is achieved with the assumption that the relative model error is less than one. The transient response of the suggested ILC algorithm is also discussed using a simulation example.
\end{abstract}

Keywords: Iterative learning control, disturbance rejection, discrete time systems, convergence 


\title{
Disturbance rejection using an ILC algorithm with iteration varying filters
}

\author{
M. Norrlöf \\ Department of Electrical Engineering, Linköping University, \\ SE-581 83 Linköping, Sweden \\ Email: mino@isy.liu.se, phone: +46 13282704, fax: +46 13282622
}

\section{Introduction}

In this paper it is shown how an Iterative Learning Control (ILC) algorithm with iteration varying filter gain can be found using ideas from estimation and identification theory [10]. Iterative learning control is a widely spread method for achieving trajectory tracking [3] (see also e.g., the surveys $[13,14,4,11]$ ). Here ILC will instead be used in a different application, namely for disturbance rejection. Disturbance rejection aspects of ILC have been covered previously in e.g., [18, 6, 5, 9, 20], where disturbances such as initial state disturbances and measurement disturbances are addressed. In [20] and [21] a stochastic ILC algorithm is presented and applied to a system when a system disturbance is acting on the input. One result from [21] is that the convergence rate of the input-variance of to the system is inversely proportional to the iteration number $k$. A similar result is derived here for the ILC filter gain and the output variance of the system. An important result is also that asymptotically the output disturbance is completely compensated for and the variance of the controlled variable goes to zero as long as the relative model error is less than 1 .

Previous work on disturbance aspects of ILC by the author is [17] where disturbance aspects of iteration invariant ILC algorithms are dealt with. In [16] iteration varying ILC algorithms are covered and a model based scheme based upon Kalman filters [1] and LQ-design [2] techniques is developed. The gain of the Kalman filter becomes a function of iteration since an estimate of the output load disturbance variance is included in the design. The previous contributions differ from what is presented here in that [17] only deals with iteration invariant ILC algorithms and [16] is by nature an adaptive scheme.

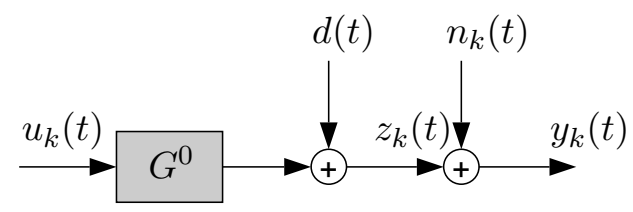

Figure 1: The system considered in the disturbance rejection approach.

In Figure 1 a block diagram representation of the disturbance rejection formulation approach to ILC is shown. In ILC the goal is to iteratively find the input to the system 
such that the error is minimized. With the disturbance rejection formulation, illustrated in Figure 1, the goal is to find an input $u_{k}(t)$ such that the output $z_{k}(t)$ is minimized. If the system is known (and invertible) and the disturbance $d(t)$ is known, then the best approach would be to filter $d(t)$ through the inverse of the system and use the resulting ${ }^{1}$ $u(t)=-\left(G^{0}(q)\right)^{-1} d(t)$ as a control input.

\section{System description}

The system $G^{0}$ in Figure 1 is assumed to be a discrete time single input single output linear time invariant (SISO LTI) system. The mathematical description becomes,

$$
\begin{aligned}
& z_{k}(t)=G^{0}(q) u_{k}(t)+d(t) \\
& y_{k}(t)=z_{k}(t)+n_{k}(t)
\end{aligned}
$$

where $u_{k}(t)$ is the input, $d(t)$ is the disturbance, $z_{k}(t)$ is the controlled variable, $n_{k}(t)$ is the measurement disturbance and, finally, $y_{k}(t)$ is the measured variable. Assume that $G(q)$ is a model of the system $G^{0}(q)$ and that

$$
G^{0}(q)=\left(1+\Delta_{G}(q)\right) G(q)
$$

where $\Delta_{G}(q)$ is a relative model uncertainty.

The measurement disturbance $n_{k}(t)$ is assumed to be defined as $n_{k}(t)=\nu(\bar{t})$ where $\bar{t}=$ $k \cdot t_{f}+t$ and $\nu(\bar{t})$ represents a white stationary stochastic process with variance $r_{n}$. The expected value, $E\left\{n_{k}(t)\right\}$, is therefore with respect to the underlying process $\nu$, and

$$
E\left\{n_{k}(t)\right\}=0
$$

The variance becomes

$$
\operatorname{Var}\left\{n_{k}(t)\right\}=r_{n}
$$

and since $\nu$ is white it implies $E\left\{n_{i}\left(t_{1}\right) n_{j}\left(t_{2}\right)\right\}=r_{n}$ if and only if $i=j$ and $t_{1}=t_{2}$ (and 0 otherwise). Note that since $d(t)$ is a deterministic signal, the expected value becomes $E\{d(t)\}=d(t)$.

\section{Disturbance estimation based ILC}

Using data from an experiment and the model of the system, the disturbance $d(t)$ at time $t$ can be estimated according to

$$
\tilde{y}_{k}(t)=y_{k}(t)-G(q) u_{k}(t)
$$

Let $\hat{d}_{k}(t)$ be the estimate of the disturbance in the $k$ th iteration computed such that the loss function

$$
V_{k, t}\left(\hat{d}_{k}(t)\right)=\frac{1}{2} \sum_{j=0}^{k-1}\left(\tilde{y}_{j}(t)-\hat{d}_{k}(t)\right)^{2}
$$

\footnotetext{
${ }^{1} q$ is the time-shift operator $q d(t)=d(t+1)$
} 
is minimized. The corresponding estimate is given by,

$$
\hat{d}_{k}(t)=\frac{1}{k} \sum_{j=0}^{k-1} \tilde{y}_{j}(t)
$$

which can also be written in iterative form

$$
\hat{d}_{k+1}(t)=\frac{k}{k+1} \hat{d}_{k}(t)+\frac{1}{k+1} \tilde{y}_{k}(t)
$$

The corresponding ILC algorithm is an update equation for the control signal $u_{k}(t)$. In order to minimize $y_{k}(t)$ the best choice for the input is

$$
u_{k+1}(t)=-\frac{1}{G(q)} \hat{d}_{k+1}(t)
$$

which means that,

$$
u_{k+1}(t)=u_{k}(t)-\frac{1}{(k+1) G(q)} y_{k}(t)
$$

where (6), (3), and (7) have been used. Compare with the "standard" first order ILC updating equation [8],

$$
u_{k+1}(t)=Q(q)\left(u_{k}(t)+L(q) e_{k}(t)\right)
$$

where $e_{k}(t)$ is the error. In the disturbance rejection approach $e_{k}(t)$ is simply the output $y_{k}(t)$. (8) is a particular case of (9) where the $Q$-filter is chosen as $Q \equiv 1$ and the $L$-filter, $L_{k}(q)=-\frac{1}{(k+1) G(q)}$, is iteration dependent so that the gain is reduced every iteration. Notice that $L_{k}(q)$ normally is a non-causal filter but since $y_{k}(t), 0 \leq t \leq n-1$, is available when computing $u_{k+1}(t)$ this is not a problem. The fact that non-causal filters can be utilized in ILC algorithms is one reason why it is possible to achieve such good results with often quite simple filters $Q$ and $L$. This is also explored in e.g., [7] and [12].

Remark 1 The gain of the filter $L_{k}$ is reduced with $k$ and this indicates that the algorithm will not work very well if $d(t)$ is varying as a function of iteration. To be able to track an iteration varying disturbance $d_{k}(t)$ the gain of the ILC algorithm cannot tend to zero as the number of iterations increases.

The mathematical description of the system is given by (1) and the ILC updating equation by (8). It is assumed that $u_{0}(t)$ is chosen as $u_{0}(t)=0, t \in\left[0, t_{f}\right]$.

\section{Analysis}

The analysis is divided in two cases, first when the true system is known and second when only a model of the system is available.

\subsection{True system known}

Consider the estimator in (5),

$$
\hat{d}_{k}(t)=\frac{1}{k} \sum_{j=0}^{k-1}\left(y_{k}(t)-G(q) u_{k}(t)\right)
$$


When the system is known, i.e., $G(q)=G^{0}(q)$, and the disturbance $n_{k}(t)$ is defined as in Section 2, then the estimator will asymptotically give an unbiased estimate of the disturbance $d(t)$,

$$
\lim _{k \rightarrow \infty} \hat{d}_{k}(t)=\lim _{k \rightarrow \infty} \frac{1}{k} \sum_{j=0}^{k-1}\left(d(t)+n_{j}(t)\right)=d(t) .
$$

If the measurement noise is assumed to have a mean different from zero, say $E\left\{n_{k}(t)\right\}=\bar{n}$, then the estimate in (10) will be biased,

$$
\lim _{k \rightarrow \infty} \hat{d}_{k}(t)=d(t)+\bar{n}
$$

From the ILC perspective an unbiased estimate implies that the algorithm will converge to zero error while a biased estimate will cause a final error. Obviously, it is not only the fact that the estimate is unbiased that is of interest. The variance of the estimate is also an important property. The variance is given by,

$$
\begin{aligned}
& \operatorname{Var}\left(\hat{d}_{k}(t)\right)=E\left\{\hat{d}_{k}^{2}(t)\right\}-\left(E\left\{\hat{d}_{k}(t)\right\}\right)^{2}= \\
& E\left\{\frac{1}{k^{2}} \sum_{i=0}^{k-1}\left(d(t)+n_{i}(t)\right) \sum_{j=0}^{k-1}\left(d(t)+n_{j}(t)\right)\right\}-d^{2}(t)= \\
& \quad \frac{1}{k^{2}} \sum_{i=0}^{k-1} \sum_{j=0}^{k-1} E\left\{d^{2}(t)+d(t)\left(n_{i}(t)+n_{j}(t)\right)\right. \\
& \left.\quad+n_{i}(t) n_{j}(t)\right\}-d^{2}(t)=\frac{r_{n}}{k}
\end{aligned}
$$

where the last equality follows from the fact that $d(t)$ is deterministic, $E\left\{n_{i}(t)\right\}=0$, and that $E\left\{n_{i}(t) n_{j}(t)\right\}=r_{n}$ if $i=j$ and 0 otherwise, see also Section 2 .

Remark 2 If the mean of the measurement disturbance is different from zero there will be a number of extra terms in (12) but the resulting variance is the same.

It is also important to study how the resulting control input, $u_{k}(t)$, develops. Using the updating equation in (8) with the true system $G^{0}(q)$ instead of $G(q)$ and $u_{0}(t)=0$ the controlled variable $z_{1}(t)$ becomes,

$$
\begin{aligned}
z_{1}(t) & =G^{0}(q) u_{1}(t)+d(t) \\
& =-G^{0}(q) \frac{1}{G^{0}(q)}\left(d(t)+n_{0}(t)\right)+d(t)=-n_{0}(t) .
\end{aligned}
$$

This means that $d(t)$ is completely compensated for and the mathematical expectation of $z_{1}(t)$ is zero. What can be improved is however the variance of $z_{k}(t)$. It is easily calculated as

$$
\operatorname{Var}\left(z_{1}(t)\right)=r_{n}
$$

The best result that can be achieved is when the disturbance $d(t)$ is perfectly known. This gives $z(t)=G^{0}(q) \frac{1}{G^{0}(q)} d(t)-d(t)=0$ i.e., zero variance. The proposed algorithm (8) is evaluated in a simulation. 
Example 1 The system $G^{0}(q)=\frac{0.07 q^{-1}}{1-0.93 q^{-1}}$ is assumed known, $r_{n}=1$, $\sum_{t \in\left[0, t_{f}\right]} d^{2}(t)=2(N-1)$, and the normalized estimated variance of $z_{k}$,

$$
V_{k}=\frac{1}{r_{n}} \cdot \frac{1}{N-1} \sum_{t \in\left[0, t_{f}\right]} z_{k}^{2}(t)
$$

is utilized as performance measure. $N$ is the number of samples in the set $\left[0, \ldots, t_{f}\right]$. From (14) it is clear that $V_{1}=1$ which is also shown in Figure 2. Notice that $V_{0}$ does not correspond to a variance since $z_{0}(t)=d(t)$. $V_{0}$ therefore depends only on the size of the disturbance $d(t)$. The simulation is however done to show what happens with the variance of $z_{k}(t)$ when $k \geq 1$.

$A$ rapid decrease of $V_{k}$ can be seen in the first iterations. After 10 iterations, for example, $V_{k}$ is reduced to 0.1 . To reduce the last 0.1 units down to 0 , however, it takes infinitely many iterations.

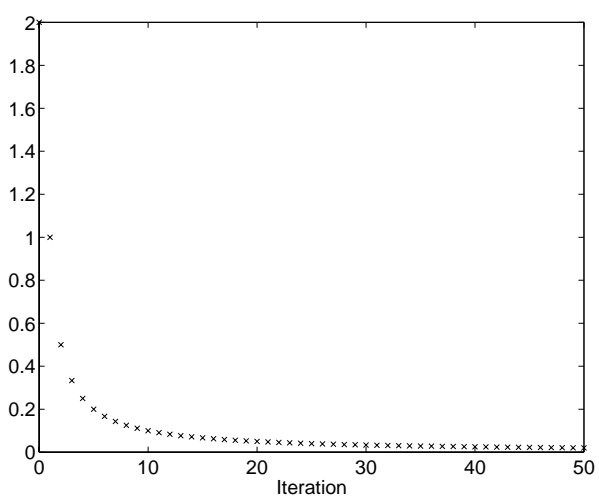

Figure 2: Evaluation of $V_{k}$ from (15) in a simulation.

The conclusion from this simulation is that the use of the proposed ILC algorithm gives a reduced variance of the controlled variable when the system is completely known but the disturbance is unknown. In the next section the properties of the method will be examined when the system is not completely known.

\subsection{True system unknown}

In practice it is clear that a model of the true system has to be used in the ILC algorithm. In this section some general results will be found relating the model uncertainty to the performance of the ILC algorithm. As mentioned in Section 2 (2) it is reasonable to assume that a model of the system is known with some model error $\Delta_{G}$. The following general ILC algorithm

$$
u_{k+1}(t)=u_{k}(t)-L_{k}(q) y_{k}(t)
$$


is used and $L_{k}(q)$ is assumed to be chosen according to

$$
\begin{aligned}
L_{k}(q) & =\frac{1}{G(q)} \\
L_{k}(q) & =\frac{1}{(k+1) G(q)}
\end{aligned}
$$

Using the ILC updating scheme in (16) and the filter in (17), it is straightforward to arrive at

$$
\begin{aligned}
z_{1}(t) & =-\Delta_{G}(q) d(t)-\left(1+\Delta_{G}(q)\right) n_{0}(t) \\
& =-\Delta_{G}(q) z_{0}(t)-\left(1+\Delta_{G}(q)\right) n_{0}(t)
\end{aligned}
$$

and in general

$$
z_{k+1}(t)=-\Delta_{G}(q) z_{k}(t)-\left(1+\Delta_{G}(q)\right) n_{k}(t)
$$

This can be expressed by the following finite sum

$$
z_{k+1}(t)=-\left(1+\Delta_{G}(q)\right) \sum_{j=0}^{k}\left(-\Delta_{G}(q)\right)^{j} n_{k-j}(t)+\left(-\Delta_{G}(q)\right)^{k+1} d(t)
$$

Remark 3 Clearly (19) and (20) are valid also when there is no model error, i.e., $\Delta_{G}(q) \equiv$ 0 . When $\Delta_{G}(q)=0$ the controlled variable becomes $z_{k+1}(t)=n_{k}(t), z_{0}(t)=d(t)$ and $\operatorname{Var}\left(z_{k}(t)\right)=r_{n}$.

Remark 4 Notice that a sufficient stability condition for the ILC algorithm in (16) with $L_{k}(q)$ from (17) is that $\left\|\Delta_{G}\right\|_{\infty}<1$ which can be interpreted as "the model can have an error of $100 \%$ but still give a stable ILC algorithm" (see also [12]).

In a deterministic framework (20) is simply $z_{k+1}=\left(-\Delta_{G}(q)\right)^{k+1} d(t)$ and if there are no measurement disturbances acting on the system the error will converge to zero if the relative model error is less than $100 \%\left(\left\|\Delta_{G}\right\|_{\infty}<1\right)$. This is one reason why ILC has been so successful in applications. The results on stability and convergence in time and frequency domain are treated in more detail in e.g., [15].

When the filter from (18) is considered and the true system is not known then the equation corresponding to (19) becomes

$$
z_{k+1}(t)=\frac{k-\Delta_{G}(q)}{k+1} z_{k}(t)-\frac{1+\Delta_{G}(q)}{k+1} n_{k}(t)
$$

with $z_{0}(t)=d(t)$. As in (19) and (20) it is possible to express (21) in an explicit form,

$$
\begin{aligned}
z_{k+1}(t)=\prod_{i=0}^{k} \frac{i-\Delta_{G}}{i+1} d(t) & -\frac{1+\Delta_{G}}{k+1} n_{k}(t) \\
& -\underbrace{\sum_{i=0}^{k-1} \prod_{j=i+1}^{k} \frac{j-\Delta_{G}}{j+1} \cdot \frac{1+\Delta_{G}}{i+1} n_{i}(t)}_{=0 \text { if } k=0}
\end{aligned}
$$

Next a general stability result for the model based ILC algorithm with iteration varying filters is presented. 
Theorem 1 (Stability with model uncertainty) The ILC algorithm from (16) with the $L_{k}$ filter in (18) converges to zero error and zero variance,

$$
\lim _{k \rightarrow \infty} E\left\{z_{k}(t)\right\}=0, \quad \lim _{k \rightarrow \infty} \operatorname{Var}\left\{z_{k}(t)\right\}=0
$$

if $\left\|\Delta_{G}\right\|_{\infty}<1$.

Proof. First it is shown that the effect of $d(t)$ is removed when $k \rightarrow \infty$. Consider the first term in (22). Using the norm to get an upper bound gives

$$
\lim _{k \rightarrow \infty} \prod_{i=0}^{k} \frac{i-\Delta_{G}}{i+1} d(t) \leq \lim _{k \rightarrow \infty} \prod_{i=0}^{k} \frac{i+\left\|\Delta_{G}\right\|_{\infty}}{i+1} d(t)=0
$$

The last equality follows from (see e.g., [19])

$$
\lim _{k \rightarrow \infty} \prod_{i=2}^{k}\left(1-\frac{1}{i}\right)=0
$$

and that there exists a $j \in \mathbb{Z}^{+}$s.t. $\frac{1}{j}<1-\left\|\Delta_{G}\right\|_{\infty}$, and hence

$$
\lim _{k \rightarrow \infty} \prod_{i=1}^{k+1}\left(1-\frac{1-\left\|\Delta_{G}\right\|_{\infty}}{i}\right) \leq \frac{\lim _{k \rightarrow \infty} \prod_{i=2}^{k+j}\left(1-\frac{1}{i}\right)}{\prod_{i=2}^{j-1}\left(1-\frac{1}{i}\right)}=0
$$

The variance expression is found from

$$
z_{k+1}(t)=\frac{k-\Delta_{G}(q)}{k+1} z_{k}(t)-\frac{1+\Delta_{G}(q)}{k+1} n_{k}(t)
$$

Clearly $\frac{k-\Delta_{G}(q)}{k+1} \leq \frac{k+\left\|\Delta_{G}\right\|_{\infty}}{k+1}<1$ when $\left\|\Delta_{G}\right\|_{\infty}<1$ and the second term $\rightarrow 0$ when $k \rightarrow \infty$. This implies that

$$
\lim _{k \rightarrow \infty} z_{k+1}(t)-z_{k}(t)=0
$$

and hence

$$
\lim _{k \rightarrow \infty} \operatorname{Var}\left(z_{k}\right)=0 .
$$

Theorem 1 shows that when the model error is less than $100 \%$ the disturbance will asymptotically be completely compensated for. The rate of convergence will be discussed in the next section. 


\section{Simulation results}

Consider the ILC updating scheme in (8) applied to the system in (1). The filters $L_{k}(q)$ are chosen as

$$
\begin{aligned}
& L_{k}(q)=\left(G^{0}(q)\right)^{-1} \\
& L_{k}(q)=\mu \cdot\left(G^{0}(q)\right)^{-1} \\
& L_{k}(q)=(G(q))^{-1} \\
& L_{k}(q)=\frac{1}{k+1}\left(G^{0}(q)\right)^{-1} \\
& L_{k}(q)=\frac{1}{k+1}(G(q))^{-1}
\end{aligned}
$$

The system $G^{0}$ is given by

$$
G^{0}(q)=\frac{0.07 q^{-1}}{1-0.93 q^{-1}}
$$

and the model $G$ by

$$
G(q)=\frac{0.15 q^{-1}}{1-0.9 q^{-1}}
$$

The ILC updating scheme in (8) gives a stable ILC system for all the different choices of $L_{k}$-filters in (23). With $G^{0}$ and $G$ according to $(24)$ and $(25)\left\|\Delta_{G}\right\|_{\infty}=0.541(<1)$. To compare the transient behavior of the five ILC schemes created by using the updating scheme from (8) and the filters from (23) a simulation is performed. The system used in the simulation is given by (1) with the actual system description (24). The model of the system, available for the ILC control scheme, is given by (25). The variance of the additive noise, $n_{k}(t)$, is set to $10^{-3}$.

To evaluate the results from the simulations the following measure is used

$$
V\left(z_{k}\right)=\frac{1}{n-1} \sum_{t=1}^{n} z_{k}^{2}(t)
$$

which is an estimate of the variance if $z_{k}$ is a random variable with zero mean. In Figure 3 the results from the simulations are shown. In the first iteration $V\left(z_{0}\right)$ contains only the value of $V(d)$ and for the $d$ used in the simulations $V(d)=0.179$. Obviously the ILC schemes, (23a) and (23d), give similar results in iteration 1 since both use the inverse of the true system to find the next control input. The pair, (23c) and (23e), give for the same reason similar results after one iteration. With the $L_{k}$-filter from $(23 \mathrm{~b})$ having $\mu=0.25$ the error reduces less rapidly than all the other choices of filters because of the low gain of the $L$-filter.

Figure 3 shows the general behavior that can be expected from the different ILC approaches covered by (8) and (23). It is clear that among the methods described here the approach given by (23d) is the best choice, although this method requires that the system description is completely known. If the system is not known as in (23e) the result may not be so good, cf. Figure 3 .

For the asymptotic analysis consider $L_{k}$ chosen according to (23a). Since $u_{0}(t)=0$, this means that $z_{0}(t)=d(t)$. From (1) it now follows that

$$
y_{0}(t)=d(t)+n_{k}(t)
$$


and therefore

$$
u_{1}(t)=-\left(G^{0}\right)^{-1}\left(d(t)+n_{0}(t)\right)
$$

The corresponding $z_{1}(t)$ becomes $z_{1}(t)=-n_{0}(t)$. This means that

$$
u_{2}(t)=-\left(G^{0}\right)^{-1}\left(d(t)+n_{1}(t)\right)
$$

and $z_{2}(t)=-n_{1}(t)$. Therefore the asymptotic value of $V\left(z_{k}\right)$ for $k>0$ therefore becomes equal to $r_{n}$ since with this approach $z_{k}(t)=-n_{k-1}(t), k>0$.

A more general case is when a model of the system $G^{0}$ is used in the $L$-filter. This corresponds to the choice of filter for the ILC algorithm according to (23c). Obviously $z_{0}(t)=d(t)$ also in this case. Now assume that the relation between the true system and the model can be described according to (2).

To understand why, in this case, using a model of the system gives better asymptotic performance compared to using the true system, consider (19) and (20). If $\left\|\Delta_{G}\right\|<1$ then, for a large enough $k$, the influence of $d(t)$ can be neglected, since $\left\|\Delta_{G}\right\|^{k}$ becomes small. Now assume that the model uncertainty is mainly a scaling error, i.e., the dynamics are captured by the model. This means that $\Delta_{G}(q) \approx \delta$ for some $\delta$, with $|\delta|<1$. Since the effect of $d(t)$ in $z_{k}(t)$ can be neglected for large values of $k$ the expected value of $z_{k}(t)$ becomes equal to 0 . The variance expression is found using, e.g., (19) and

$$
r_{z, k+1}=E\left\{z_{k+1}^{2}(t)\right\} \approx \delta^{2} r_{z, k}+(1+\delta)^{2} r_{n}
$$

Asymptotically this means that

$$
r_{z, \infty} \approx r_{n} \cdot \frac{1+\delta}{1-\delta}
$$

In the example when $L_{k}(q)$ from $(23 \mathrm{c})$ is used, $\delta \approx-\frac{1}{2}$ for high frequencies and using the result in (28) it follows that $r_{z, \infty} \approx \frac{r_{n}}{3}$, i.e., $r_{z, \infty} \approx 0.3 \cdot 10^{-3}$. In fact this is also what is shown in Figure 3. The conclusion from this is that it is possible to get $V\left(z_{k}\right)<r_{n}$ asymptotically by choosing a model such that $G^{0}(q)=\kappa G(q)$ for some $0<\kappa<1$ and let $L(q)=(G(q))^{-1}$. This shows why it is, in this case, better to use a model of the system. This is also shown explicitly when (23b) is applied since there the learning filter is chosen as a constant, $\mu=0.25$ times the true system. After 100 iterations this approach gives the second best level of $V_{k}$. By comparing the result using (23b) and (23c) it also becomes clear that the reduced $V\left(z_{k}\right)$ is achieved by reducing the transient performance. When using an iteration invariant learning filter it is therefore a balance between achieving a low asymptotic level of $V\left(z_{k}\right)$ and having a rapid reduction of $V\left(z_{k}\right)$ in the first iterations.

When the true model is known and used in the filter according to $(23 \mathrm{~d})$, then the value of $V\left(z_{k}\right)$ becomes equal to $\frac{r_{n}}{k}$ for $k>0$. For $k=0, V\left(z_{0}\right)$ is equal to $V(d)$ since $z_{0}=d$. If the true system is not known and the model based approach in (23e) is used, then the equation corresponding to (19) becomes $(21)$ with $z_{0}(t)=d(t)$. In Theorem 1 it was shown that as long as $\left\|\Delta_{G}\right\|_{\infty}<1, z_{k}$ will go to zero. Figure 3 shows that this method does not always give a good transient behavior. This depends on the fact that the disturbance $d(t)$ is not completely compensated for in the first iteration. Since the gain is decreased at every iteration the amount of the disturbance, $d(t)$, that will be compensated for will decrease in every iteration. This means that instead of being dominated by the random disturbance the measure $V(z)$ will instead be dominated by a term depending on the disturbance $d(t)$. Although this part will eventually will be compensated for it can converge very slow if $\left\|\Delta_{G}\right\|_{\infty}$ is close to 1 . 

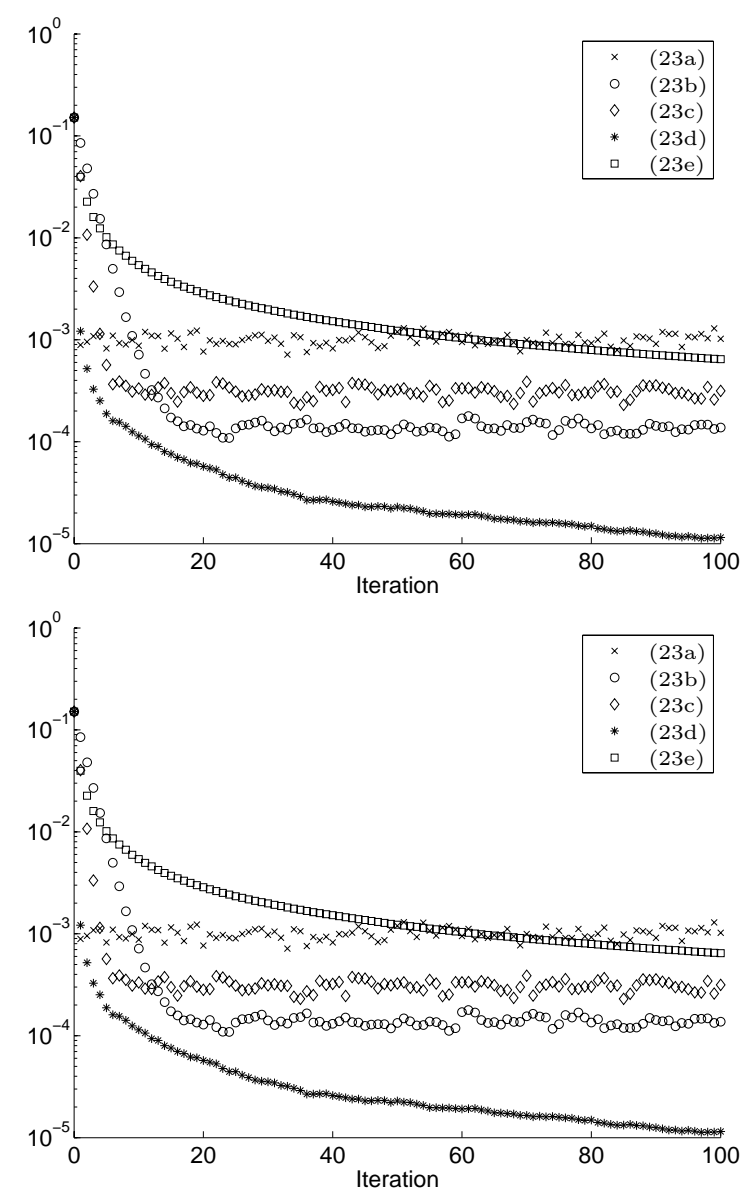

Figure 3: The transient behavior of $V_{k}$ for the 5 different ILC schemes given by (8) and (23). Iterations 0 to 100 are shown in the upper figure and a zoom of iterations 0 to 20 is shown in the lower figure. 


\section{Conclusion}

The major contribution of this paper is to show that when introducing a measurement disturbance together with iterative learning control and taking this disturbance into account the filters in the ILC algorithm become iteration variant. If the relative model error $\Delta_{G}$ from (2) satisfies $\left\|\Delta_{G}\right\|_{\infty}<1$ then the disturbance $d(t)$ will be asymptotically completely compensated for and the variance of the controlled variable $z_{k}(t)$ will be equal to zero. The ILC algorithm with iteration varying filters was found using ideas from estimation theory. A similar result although not derived in the same framework can be found in [20] and [21]. When using iteration invariant filters the gain of the $L$-filter becomes important and by adjusting this gain it is possible to reach a lower norm of the asymptotic error than just applying the inverse system model as a learning filter.

\section{Acknowledgments}

The author would like to thank the anonymous reviewers for giving constructive comments on the first version of the paper. The comments have significantly improved the mathematical results and the presentation. This work was supported by the VINNOVA Center of Excellence ISIS at Linköping University, Linköping, Sweden.

\section{References}

[1] Brian D.O. Anderson and John B. Moore. Optimal Filtering. Prentice-Hall, 1979.

[2] Brian D.O. Anderson and John B. Moore. Optimal Control: Linear Quadratic Methods. Prentice-Hall, 1989.

[3] S. Arimoto, S. Kawamura, and F. Miyazaki. Bettering operation of dynamic systems by learning: A new control theory for servomechanism or mechatronics systems. In Proc. of the 23rd IEEE Conf. on Decision and Control, pages 1064-1069, Las Vegas, NV, Dec 1984.

[4] Z. Bien and J.-X. Xu. Iterative Learning Control: Analysis, Design, Integration and Application. Kluwer Academic Publishers, Boston, MA, 1998.

[5] Y. Chen, C. Wen, J.-X. Xu, and M. Sun. An initial state learning method for iterative learning control of uncertain time-varying systems. In Proc. of the 35th Conf. on Decision and Control, pages 3996-4001, Kobe, Japan, Dec 1996.

[6] C.-J. Chien. A discrete iterative learning control of nonlinear time-varying systems. In Proc. of the 35th IEEE Conf. on Decision and Control, pages 3056-3061, Kobe, Japan, Dec 1996.

[7] Peter B. Goldsmith. On the equivalence of causal lti iterative learning control and feedback control. Automatica, 38:703-708, April 2002.

[8] S. Hara, Y. Yamamoto, T. Omata, and M. Nakano. Repetitive control system: A new type servo system for periodic exogenous signals. IEEE Transactions on Automatic Control, 33(7):659-668, 1988. 
[9] G. Heinzinger, D. Fenwick, B. Paden, and F. Miyazaki. Stability of learning control with disturbances and uncertain initial conditions. IEEE Transactions on Automatic Control, 37(1):110-114, Jan 1992.

[10] L. Ljung. Model error modeling and control design. In The IFAC Symposium on System Identification, SYSID2000, 2000.

[11] R.W. Longman. Iterative learning control and repetitive control for engineering practice. International Journal of Control, 73(10):930 - 954, July 2000.

[12] O. Markusson, H. Hjalmarsson, and M. Norrlöf. A general framework for iterative learning control. In Proceedings of the 15th IFAC World Congress, Barcelona, Spain, July 2002.

[13] K. L. Moore. Iterative Learning Control for Deterministic Systems. Advances in Industrial Control. Springer-Verlag, London, 1993.

[14] K. L. Moore. Iterative learning control - an expository overview. Applied and Computational Controls, Signal Processing and Circuits, 1:151-214, 1999.

[15] M. Norrlöf and S. Gunnarsson. Time and frequency domain convergence properties in iterative learning control. International Journal of Control, 75(14):1114-1126, 2002.

[16] M. Norrlöf. An adaptive iterative learning control algorithm with experiments on an industrial robot. IEEE Transactions on Robotics and Automation, 18(2):245-251, April 2002.

[17] M. Norrlöf and S. Gunnarsson. Disturbance aspects of iterative learning control. Engineering Applications of Artificial Intelligence, 14(1):87-94, 2001.

[18] S. Panzieri and G. Ulivi. Disturbance rejection of iterative learning control applied to trajectory tracking for a flexible manipulator. In Proceedings of the 3rd European Control Conference, pages 2374-2379, Sep 1995.

[19] A.P. Prudnikov. Integrals and series. Vol. 1, Elementary functions. Gordon and Breach, New York, 1986.

[20] S.S. Saab. A discrete-time stochastic learning control algorithm. IEEE Transactions on Automatic Control, 46(6):877-887, June 2001.

[21] S.S. Saab. On a discrete-time stochastic learning control algorithm. IEEE Transactions on Automatic Control, 46(8):1333-1336, Aug 2001. 


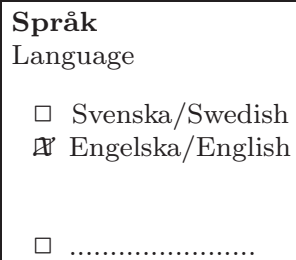

URL för elektronisk version

http://www. control.isy.liu.se

\section{Titel}

Title

Författare

Author

$$
\text { Mikael Norrlöf, }
$$

Sammanfattning

Abstract

An Iterative Learning Control disturbance rejection approach is considered and it is shown that iteration variant learning filters can asymptotically give the controlled variable zero error and zero variance. Convergence is achieved with the assumption that the relative model error is less than one. The transient response of the suggested ILC algorithm is also discussed using a simulation example.

Nyckelord

Keywords

Iterative learning control, disturbance rejection, discrete time systems, convergence

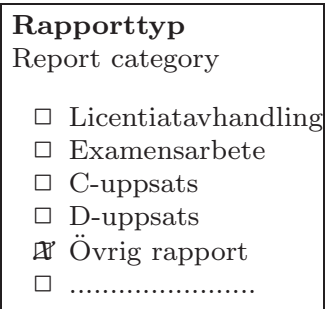

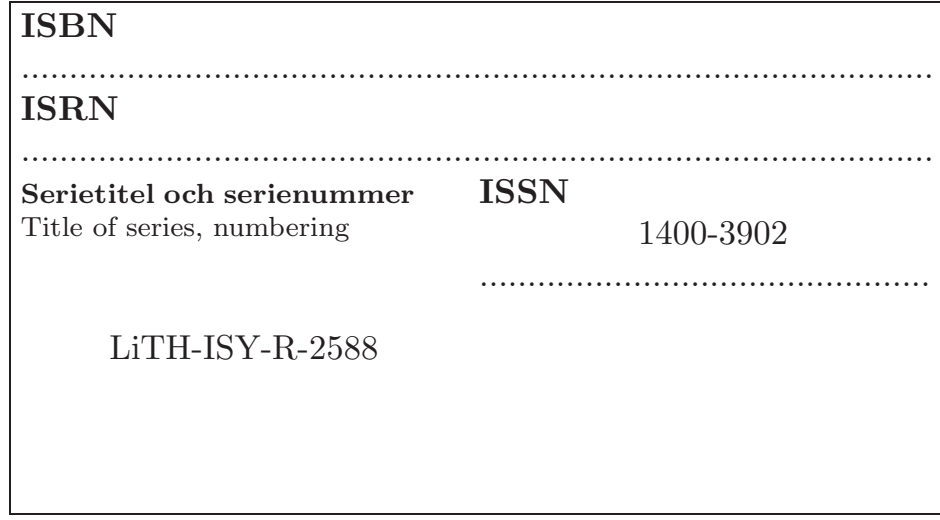

Disturbance rejection using an ILC algorithm with iteration varying filters 